\title{
Therapeutic Outcome of Alemtuzumab in Korean Patients with Multiple Sclerosis: 2-Year Follow-Up
}

\author{
Jae-Won Hyun \\ Hyun-June Shin \\ Hyunmin Jang \\ Na Young Park \\ Su-Hyun Kim \\ Ho Jin Kim
}

Department of Neurology,

Research Institute and

Hospital of National Cancer Center, Goyang, Korea
Received November 5, 2018

Revised January 192019

Accepted January 22, 2019

\section{Correspondence}

Ho Jin Kim, MD, PhD

Department of Neurology,

Research Institute and

Hospital of National Cancer Center,

323 Ilsan-ro, Ilsandong-gu,

Goyang 10408, Korea

Tel +82-31-920-2438

Fax +82-31-905-5524

E-mail hojinkim@ncc.re.kr
Background and Purpose Alemtuzumab has shown high efficacy in clinical trials that primarily involved Western multiple sclerosis (MS) patients. To evaluate the therapeutic outcome of alemtuzumab in Korean patients with MS.

Methods This study enrolled 23 consecutive patients who were treated with alemtuzumab from 2015 to 2018. Efficacy was evaluated using the annualized relapse rate (ARR), Expanded Disability Status Scale (EDSS), and radiological activity. No evidence of disease activity (NEDA) was defined as no clinical relapse, no worsening of the EDSS score, and no radiological activity. The safety profiles were also assessed.

Results The mean age was 36 years and 16 of the patients were female. Seventeen and 12 of 23 patients were followed up for 1 year and 2 years, respectively. The ARR was markedly reduced from 1.52 during the 1-year period preceding alemtuzumab administration to 0.21 after initiating alemtuzumab $(p<0.001)$. During the first and second years after initiating alemtuzumab, EDSS worsening was observed in $3(18 \%)$ and $0(0 \%)$ patients, respectively, and radiological activity was exhibited in $9(53 \%)$ and $4(33 \%)$. NEDA was observed in $6(35 \%)$ patients during the first year and in 8 (67\%) patients during the second year. Intriguingly, one patient experienced 2 severe clinical exacerbations, which occurred at 10 months after the first and 10 months after the second infusion of alemtuzumab. Nineteen of the 23 patients exhibited infusion-associated reactions and 3 patients exhibited herpes zoster infection. Thyroid dysfunction occurred in two patients at 18 and 20 months after initiating alemtuzumab.

Conclusions Consistent with observations in Western populations, alemtuzumab therapy in Korean MS patients led to marked reductions of disease activity without unexpected safety issues.

Key Words multiple sclerosis, alemtuzumab, efficacy, safety.

\section{INTRODUCTION}

Alemtuzumab is an anti-CD52 monoclonal antibody that depletes and repopulates circulating $\mathrm{T}$ and $\mathrm{B}$ lymphocytes, thereby shifting cytokine expression toward a less-inflammatory condition. ${ }^{1}$ Alemtuzumab was shown to be a highly efficacious therapy with tolerable safety in previous studies-including clinical trials-that mainly involved Western patients with multiple sclerosis (MS) ${ }^{2-11}$ The prevalence of MS is much higher in Western populations than in Asian populations, and various disease-modifying therapies (DMTs) for MS have been approved later in Asian countries than in Western countries. ${ }^{12,13}$ In order to broaden the use of alemtuzumab as an efficacious and tolerable therapeutic option for patients with active MS, the therapeutic outcomes of alemtuzumab need to be comprehensively evaluated in various racial groups.

Alemtuzumab was approved for use in Korea in 2015, and was reimbursed by the gov-

(a) This is an Open Access article distributed under the terms of the Creative Commons Attribution Non-Commercial License (https://creativecommons.org/licenses/by-nc/4.0) which permits unrestricted non-commercial use, distribution, and reproduction in any medium, provided the original work is properly cited. 
ernment as a second-line DMT after treatment failure of first-line DMTs starting in December 2015. ${ }^{13}$ The administration of alemtuzumab basically consists of two courses of infusion, 1 year apart. ${ }^{14}$ By 2018, a subset of the patients in our clinic could be reached for 2 years of follow-up after initiating alemtuzumab. In this study we aimed to elucidate the efficacy and safety profiles of alemtuzumab in Korean patients with active MS.

\section{METHODS}

\section{Patients}

This study enrolled 23 consecutive patients with highly active relapsing MS who had been diagnosed using the 2010 McDonald criteria and treated with alemtuzumab at the National Cancer Center (NCC) in Korea between 2015 and 2018. ${ }^{15}$ Alemtuzumab was administered at $12 \mathrm{mg}$ daily at baseline (for 5 consecutive days) and 1 year later (for 3 consecutive days). ${ }^{14}$ Prophylactic intravenous methylprednisolone (IVMP; $1 \mathrm{~g}$ daily for 3 days) was started immediately before the infusion of alemtuzumab, and oral herpes prophylactic agents were started upon the initiation of alemtuzumab and continued for at least 1 month following the last alemtuzumab infusion. ${ }^{14}$ All of the patients had exhibited radiological and/or clinical disease activity at 1 year preceding the initiation of alemtuzumab despite the use of first-line DMTs. The Institutional Review Board of the NCC approved this study protocol (approval no. NCC 2014-0146) and written informed consent was obtained from all of the enrolled patients.

\section{Efficacy}

Expanded Disability Status Scale (EDSS) scores and brain MRI scans were evaluated prospectively every 3 and 6 months, respectively. Clinical relapse was defined as a clinical exacerbation with objective signs on a neurological examination that lasted at least 48 hours and was confirmed by a neurologist. ${ }^{5,6}$ At the time when clinical relapse was diagnosed, EDSS scores and brain and/or spinal cord MRI scans were also assessed according to the presence of clinical symptoms.

The efficacy was investigated clinically and radiologically. The clinical assessment involved an evaluation using the annualized relapse rate (ARR) and changes in EDSS scores. Disability worsening was defined as an increase of $\geq 1.0$ point if the baseline EDSS score was $>0$, or an increase of $\geq 1.5$ points if the baseline EDSS score was 0; disability improvement was defined as a decrease of $\geq 1.0$ point in patients with a baseline EDSS score of $\geq 2.0$. Radiological assessment was performed using brain MRI, including the presence of new or enlargement of T2-weighted lesions or gadolinium-enhanced T1weighted lesions. ${ }^{5,6}$ No evidence of disease activity (NEDA) was defined as no clinical relapse, no worsening of the EDSS score, and no radiological activity.,

\section{Safety}

Adverse events and medical events of interest were investigated. Infusion-associated reactions (IARs) were defined as adverse events that occurred during the initiation of the infusion or within 1 day postinfusion. ${ }^{5,6}$ Laboratory tests for hematology, serum creatinine, and urinalysis with microscopy were performed monthly. Thyroid function tests were performed at least every 3 months.

\section{Statistical analysis}

The Wilcoxon signed-rank test and paired $t$-test were used to compare the ARRs and EDSS scores between before and after administering alemtuzumab, with a probability value of $p<$ 0.05 considered statistically significant.

\section{RESULTS}

\section{Demographics}

The mean age of the 23 patients with MS was 36 years and their female:male ratio was 16:7. Seventeen and 12 of the 23 patients reached the 1-year and 2-year follow-ups after initiating alemtuzumab, respectively. The second course of alemtuzumab in the second year of initiation was administered in 16 patients; 1 patient refused the infusion of the second course of alemtuzumab because of EDSS scores worsening despite receiving alemtuzumab. The median baseline EDSS score was 4.0 (range 0-7.5), the mean disease duration was 9.7 years (range 2-20 years), and the mean follow-up duration after initiating alemtuzumab was 17 months (range 1-24 months). The mean number of previous DMTs before initiating alemtuzumab was 1.8 (range 1-4).

\section{Efficacy}

The mean ARR decreased markedly from 1.52 during the 1 -year period preceding the alemtuzumab administration to 0.21 after initiating alemtuzumab $(p<0.001)$, indicating a $86 \%$ risk reduction (Fig. 1). At the completion of 2 years of follow-up after commencement of alemtuzumab, 9 (75\%) of the 12 patients were relapse-free. Five clinical relapses were observed in the other 3 patients, and as we reported previously, ${ }^{16}$ one clinical relapse (EDSS score at nadir of 9.5) was severe to require additional plasma exchanges after IVMP ( $1 \mathrm{~g}$ daily for 5 days). Three clinical exacerbations (EDSS scores at nadir of 4.0-7.0) were treated with IVMP alone, and one minor relapse recovered without IVMP treatment.

At the completion of 2 years of follow-up after initiating of alemtuzumab in 12 patients, the EDSS score had improved 


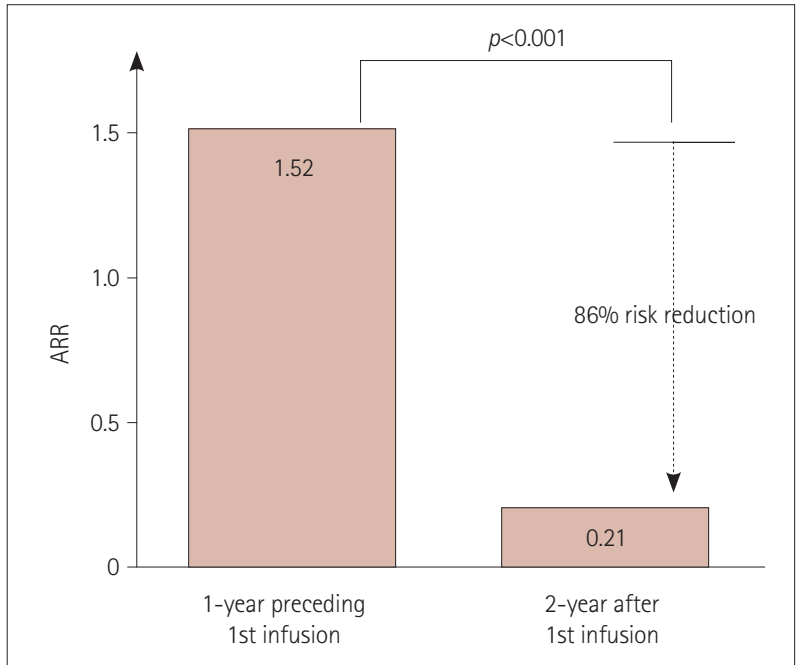

Fig. 1. One-year pretreatment and posttreatment ARRs during 2 years of follow-up in patients with multiple sclerosis receiving alemtuzumab. ARR: annualized relapse rate.

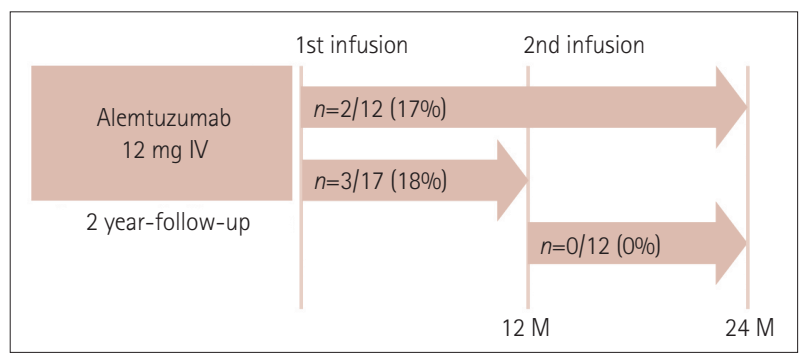

Fig. 2. Worsening of the score on the Expanded Disability Status Scale after initiating alemtuzumab during 2 years of follow-up.

in $3(25 \%)$ patients, remained stable in 7 (58\%), and worsened in 2 (17\%) (Fig. 2). During the first and second years after initiating alemtuzumab, improvements of EDSS scores were observed in 4/17 (24\%) and 1/12 (8\%) patients, respectively, stable EDSS scores were observed in 10/17 (59\%) and 11/12 (92\%) patients, and worsening of EDSS scores was observed in $3 / 17(18 \%)$ and $0 / 12(0 \%)$ patients. At the completion of 2 years of follow-up after initiating alemtuzumab, the median EDSS score had improved from 4.0 to 3.3, but this change was not statistically significant. The median EDSS score improved from 4.0 to 3.5 in 17 patients during the first year after initiating alemtuzumab and remained stable at 3.3 in 12 patients during the second year after initiating alemtuzumab.

At the completion of 2 years of follow-up after initiating alemtuzumab, radiological activity was observed in 7/12 (58\%) patients (Fig. 3). Radiological activity was observed in 9/17 (53\%) patients during the first year after initiating alemtuzumab and 4/12 (33\%) patients during the second year after initiating alemtuzumab. Radiological activity alone without clinical activity was observed in 4/12 (33\%) patients at the completion of 2 years of follow-up after initiating alem-

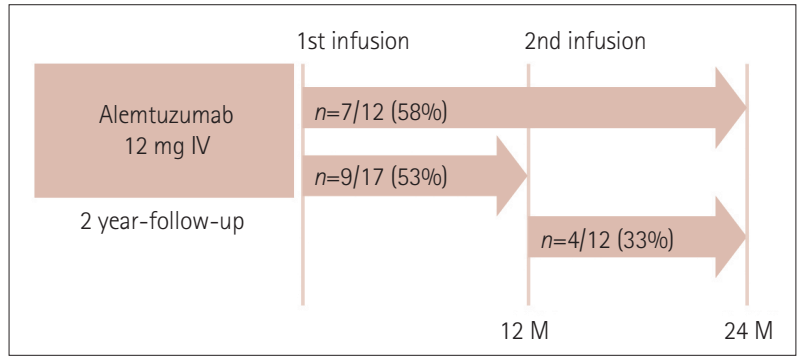

Fig. 3. Radiological activity status during 2 years of follow-up after initiating alemtuzumab.

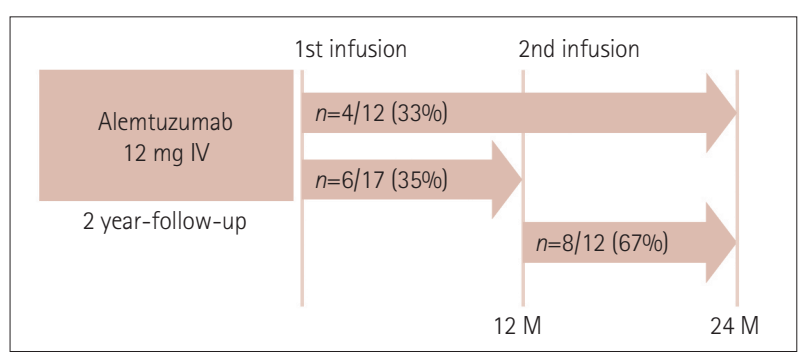

Fig. 4. No evidence of disease activity during 2 years of follow-up after initiating alemtuzumab.

tuzumab. During the first and second years after initiating alemtuzumab, 6/17 (35\%) patients and 1/12 (8\%) patient, respectively, showed radiological activity alone without clinical activity.

At the completion of 2 years of follow-up after initiating alemtuzumab, NEDA was observed in 4/12 (33\%) patients (Fig. 4). During the first and second years after initiating alemtuzumab, NEDA was observed in 6/17 (35\%) and 8/12 (67\%) patients, respectively. Three of the 4 patients who exhibited disease activity despite the second course of alemtuzumab were treated with a third course of alemtuzumab. In one patient who experienced two severe relapses (EDSS scores at nadir of 9.5 and 7.0, respectively) at 10 months after the first and 10 months after the second infusion of alemtuzumab, ${ }^{16}$ the treatment was switched to natalizumab. Two of the patients treated with a third course of alemtuzumab experienced clinical relapse with EDSS scores at nadir of 3.5 and 4.0, respectively, and the third patient exhibited only radiological activity.

\section{Safety}

Nineteen (83\%) of 23 patients exhibited IARs, including most commonly rash, urticaria, pruritis, flushing, headache, and myalgia. Table 1 lists the details of IARs in patients with MS. During the first and second courses of alemtuzumab, IARs were observed in 17/23 (74\%) and 10/16 (63\%) patients, respectively. All of these IARs were reversible, and selflimiting or controlled by symptomatic treatments (antihistamine, antipyretics, and analgesics); no patient experienced 
Table 3. Manifestations of secondary autoimmunity

Table 1. Details of infusion-associated reactions

\begin{tabular}{lcc}
\hline & $\begin{array}{c}\text { First course } \\
\text { of alemtuzumab } \\
(\boldsymbol{n}=\mathbf{2 3})\end{array}$ & $\begin{array}{c}\text { Second course } \\
\text { of alemtuzumab } \\
(\boldsymbol{n}=\mathbf{1 6})\end{array}$ \\
\hline Urticaria & 7 & 2 \\
Rash & 6 & 6 \\
Pruritis & 5 & 1 \\
Flushing & 2 & 0 \\
$\begin{array}{l}\text { Flu-like symptom } \\
\text { (myalgia or chills) }\end{array}$ & 1 & 2 \\
Headache & 0 & 2 \\
\hline
\end{tabular}

Table 2. Details of any infection

\begin{tabular}{lcc}
\hline & $\begin{array}{c}\mathbf{1} \text { year after } \\
\text { initiation }(\boldsymbol{n}=\mathbf{1 7})\end{array}$ & $\begin{array}{c}\text { 2 year after } \\
\text { initiation }(\boldsymbol{n}=\mathbf{1 2})\end{array}$ \\
\hline Herpes zoster infection & 0 & 3 \\
Urinary tract infection & 2 & 0 \\
Enteritis & 3 & 0 \\
Upper respiratory infection & 3 & 1 \\
Sinusitis & 1 & 0 \\
\hline
\end{tabular}

anaphylaxis.

Ten (43\%) of the 23 patients exhibited infection events during follow-up after initiating alemtuzumab. Three patients experienced herpes zoster infection at 20 months (two patients) or 22 months (one patient) after initiating alemtuzumab. All infection events were self-limiting, or were treated by antibiotics or antiviral agents; the details of these events are presented in Table 2.

Two patients had thyroid dysfunction, one of whom required persistent medication during follow-up (Table 3). One patient experienced hyperthyroidism at 20 months after initiating alemtuzumab, and was treated with propylthiouracil until the last follow-up; the patient's free thyroxine level had normalized after 1 month of treatment. Another patient showed a high level of thyroid-stimulating hormone combined with a low free thyroxine level at 18 months after initiating alemtuzumab; however, this was self-limiting after 6 months without applying medication. No patient experienced other manifestations of secondary autoimmunity during follow-up, including hematological or renal involvement.

\section{DISCUSSION}

The administration of alemtuzumab to Korean patients with highly active MS significantly reduced the risk of clinical relapse, and the neurological disability score tended to improve during 2 years of follow-up. NEDA was observed in 4 (33\%) of 12 patients; the proportion of patients with NEDA increased after the second course of alemtuzumab compared

\begin{tabular}{lcc}
\hline & $\begin{array}{c}\text { 1 year after } \\
\text { initiation }(\boldsymbol{n}=\mathbf{1 7})\end{array}$ & $\begin{array}{c}\text { 2 year after } \\
\text { initiation }(\boldsymbol{n}=\mathbf{1 2})\end{array}$ \\
\hline Hyperthyroidism & 0 & 1 \\
Hypothyroidism (self-limiting) & 0 & 1 \\
Idiopathic thrombocytopenia & 0 & 0 \\
Nephropathy & 0 & 0 \\
\hline
\end{tabular}

with after the first course, and reached $67 \%$ during the second year after initiating alemtuzumab. More than $80 \%$ of patients experienced IARs; however, all symptoms were manageable and no patient experienced a severe adverse event that required the discontinuation of alemtuzumab. All infection events were treatable and secondary autoimmunity developed only as thyroid dysfunction responsive to standard medication. Taken together, these results suggest that alemtuzumab is an efficacious and tolerable therapeutic option for Korean patients with highly active MS.

Patients with disease activity despite first-line DMTs might have a bad prognosis for neurological disability. ${ }^{17-19}$ Timely escalation with potent DMTs is important for reducing clinical relapse and disability progression in these patients. ${ }^{20,21}$ Prior to alemtuzumab being approved in Korea the only available second-line DMT was mitoxantrone, whose lifetime use is limited by its cumulative-dose-dependent cardiotoxicity. ${ }^{7,13}$ Alemtuzumab was approved for use as a second-line DMT in Korea at the end of 2015, and the current study has demonstrated that alemtuzumab provides a new opportunity to reduce disease activity and neurological disability accumulation in Korean patients with highly active MS.

Because all of the enrolled patients were treated with alemtuzumab as a second-line therapy in the current study, we compared the present findings with data from CARE-MS II clinical trial rather than those of CARE-MS I which enrolled only treatment-naïve patients. ${ }^{3,5}$ The proportions of patients with NEDA (33\% vs. $32 \%$ ) and improvement of disability ( $25 \%$ vs. $22 \%$ ) in the current study were comparable with CARE-MS II data from primarily Western cohorts during 2 years of follow-up after initiating alemtuzumab. ${ }^{5}$ With respect to safety issues, the incidence of IARs in the present study was comparable to that in CARE-MS $\mathrm{II}^{5,6}$ and showed a similar pattern, decreasing from $74 \%$ to $63 \%$ after the second course of alemtuzumab (from $84 \%$ to $71 \%$ in CARE-MS II). Thyroid dysfunction as a manifestation of secondary autoimmunity was observed only during the second year after initiating alemtuzumab. ${ }^{5,6}$ The peak year of thyroid dysfunction was reported at year 3 following alemtuzumab in the previous study, ${ }^{6}$ longer-term observation is needed in our cohort.

A retrospective analysis has inherent methodological lim- 
itations, although routine laboratory tests (every 1 month), EDSS scores (every 3 months), and brain MRI scans (every 6 months) were evaluated prospectively at regular intervals to monitor the treatment effect and safety. Additionally, this study enrolled only a small number $(n=23)$ of patients. However, considering the significant difference in the prevalence of MS between Western countries and Korea (67-350/100,000 persons vs. 3.5-3.6/100,000 persons), the enrolled patients could represent approximately 437 to 2,300 MS patients in Western populations. ${ }^{12,22}$ We observed comparable efficacy and safety of alemtuzumab in Korean MS patients, which represents valuable real-world evidence for the use of a new potent and tolerable therapeutic option for active MS patients in Asian populations.

Notably, one patient showed a severe clinical relapse even after administering the second course of alemtuzumab, the therapeutic regimen was subsequently changed to natalizumab. Additionally, a third course of alemtuzumab was needed in three patients who exhibited mild-to-moderate disease activity after the second course of alemtuzumab. Observations of the therapeutic outcomes in these patients and patients without an additional DMT after the second course of alemtuzumab are ongoing. In the previous extension study, 55.5\% of patients showed remission without a DMT over 3 years after the second course of alemtuzumab, and patients with disease activity received the third course of alemtuzumab and exhibited improved clinical/radiological outcomes with tolerable safety up to the 3-year follow-up. ${ }^{6,23}$ The disease course of MS can be quite heterogeneous, and personalized therapeutic approaches for individual patients are warranted. In the era of expanding therapeutic choices for patients with MS, physicians should have a comprehensive understanding of the benefit and risk profiles of each therapeutic option, and the benefit-to-risk balance should be favorably maintained to facilitate tailored therapeutic strategies for patients with MS.

In conclusion, alemtuzumab therapy in Korean patients with active MS reduced disease activity without uncontrolled safety concerns during 2 years of follow-up. An additional investigation is ongoing to assess the longer-term therapeutic outcomes of alemtuzumab therapy.

\section{Conflicts of Interest}

Shin HJ, Jang H, Park NY report no financial disclosures. Hyun JW has received a grant from the National Research Foundation of Korea. Kim SH has lectured, consulted, and received honoraria from Bayer Schering Pharma, Biogen, Genzyme, Merck Serono, and UCB and received a grant from the National Research Foundation of Korea. Kim HJ has lectured, consulted, and received honoraria from Bayer Schering Pharma, Biogen,Celltrion, Eisai, HanAll BioPharma, MedImmune, Merck Serono, Novartis, Sanofi Genzyme, Teva-Handok, and UCB; received a grant from the Ministry of Science \& ICT, Sanofi Genzyme, Teva-Handok, and UCB; and serves on a steering committee for MedImmune/VielaBio; is a co-editor for the Multiple Sclerosis Journal-Experimental, Translational, and Clinical, and an associated editor for the Journal of Clinical Neurology.

\section{Acknowledgements}

This study was supported by National Research Foundation of Korea (NRF-2017R1D1A1B03028868 \& NRF-2018R1A5A2023127), and the Bio \& Medical Technology Development Program (M3A9B6069339) through the Ministry of Science \& ICT.

\section{REFERENCES}

1. Cox AL, Thompson SA, Jones JL, Robertson VH, Hale G, Waldmann $\mathrm{H}$, et al. Lymphocyte homeostasis following therapeutic lymphocyte depletion in multiple sclerosis. Eur J Immunol 2005;35:3332-3342.

2. CAMMS223 Trial Investigators, Coles AJ, Compston DA, Selmaj KW, Lake SL, Moran S, et al. Alemtuzumab vs. interferon beta-1a in early multiple sclerosis. N Engl J Med 2008;359:1786-1801.

3. Cohen JA, Coles AJ, Arnold DL, Confavreux C, Fox EJ, Hartung HP, et al. Alemtuzumab versus interferon beta la as first-line treatment for patients with relapsing-remitting multiple sclerosis: a randomised controlled phase 3 trial. Lancet 2012;380:1819-1828.

4. Havrdova E, Arnold DL, Cohen JA, Hartung HP, Fox EJ, Giovannoni $\mathrm{G}$, et al. Alemtuzumab CARE-MS I 5-year follow-up: durable efficacy in the absence of continuous MS therapy. Neurology 2017;89:11071116.

5. Coles AJ, Twyman CL, Arnold DL, Cohen JA, Confavreux C, Fox EJ, et al. Alemtuzumab for patients with relapsing multiple sclerosis after disease-modifying therapy: a randomised controlled phase 3 trial. Lancet 2012;380:1829-1839.

6. Coles AJ, Cohen JA, Fox EJ, Giovannoni G, Hartung HP, Havrdova E, et al. Alemtuzumab CARE-MS II 5-year follow-up: efficacy and safety findings. Neurology 2017;89:1117-1126.

7. Le Page E, Deburghgraeve V, Lester MA, Cardiet I, Leray E, Edan G. Alemtuzumab as rescue therapy in a cohort of 16 aggressive multiple sclerosis patients previously treated by Mitoxantrone: an observational study. J Neurol 2015;262:1024-1034.

8. Huhn K, Bayas A, Doerck S, Frank B, Gerbershagen K, Hellwig K, et al. Alemtuzumab as rescue therapy in a cohort of 50 relapsing-remitting MS patients with breakthrough disease on fingolimod: a multicenter observational study. J Neurol 2018;265:1521-1527.

9. Prosperini L, Annovazzi P, Boffa L, Buscarinu MC, Gallo A, Matta $\mathrm{M}$, et al. No evidence of disease activity (NEDA-3) and disability improvement after alemtuzumab treatment for multiple sclerosis: a 36-month real-world study. J Neurol 2018;265:2851-2860.

10. Tuohy O, Costelloe L, Hill-Cawthorne G, Bjornson I, Harding K, Robertson $\mathrm{N}$, et al. Alemtuzumab treatment of multiple sclerosis: longterm safety and efficacy. J Neurol Neurosurg Psychiatry 2015;86:208215.

11. Willis MD, Harding KE, Pickersgill TP, Wardle M, Pearson OR, Scolding NJ, et al. Alemtuzumab for multiple sclerosis: long term follow-up in a multi-centre cohort. Mult Scler 2016;22:1215-1223.

12. Kim NH, Kim HJ, Cheong HK, Kim BJ, Lee KH, Kim EH, et al. Prevalence of multiple sclerosis in Korea. Neurology 2010;75:1432-1438.

13. Kim SH, Park MS, Kim W, Huh SY, Shin HJ, Hyun JW, et al. Realworld effectiveness of disease modifying therapies in Korean patients with relapsing multiple sclerosis. J Clin Neurol 2019;15:20-26.

14. Hartung HP, Aktas O, Boyko AN. Alemtuzumab: a new therapy for active relapsing-remitting multiple sclerosis. Mult Scler 2015;21:2234.

15. Polman CH, Reingold SC, Banwell B, Clanet M, Cohen JA, Filippi M, et al. Diagnostic criteria for multiple sclerosis: 2010 revisions to the McDonald criteria. Ann Neurol 2011;69:292-302.

16. Hyun JW, Kim Y, Kim G, Kim SH, Kim HJ. Severe B cell-mediated disease activation despite two cycles of alemtuzumab in a patient with multiple sclerosis. Mult Scler 2018 Nov 7 [Epub]. Available from: https://doi.org/10.1177/1352458518810261. 
17. Prosperini L, Gallo V, Petsas N, Borriello G, Pozzilli C. One-year MRI scan predicts clinical response to interferon beta in multiple sclerosis. Eur J Neurol 2009;16:1202-1209.

18. Rudick RA, Polman CH. Current approaches to the identification and management of breakthrough disease in patients with multiple sclerosis. Lancet Neurol 2009;8:545-559.

19. Río J, Nos C, Tintoré M, Téllez N, Galán I, Pelayo R, et al. Defining the response to interferon-beta in relapsing-remitting multiple sclerosis patients. Ann Neurol 2006;59:344-352.

20. Río J, Tintoré M, Sastre-Garriga J, Nos C, Castilló J, Tur C, et al. Change in the clinical activity of multiple sclerosis after treatment switch for suboptimal response. Eur J Neurol 2012;19:899-904.
21. Kalincik T, Horakova D, Spelman T, Jokubaitis V, Trojano M, Lugaresi A, et al. Switch to natalizumab versus fingolimod in active relapsing-remitting multiple sclerosis. Ann Neurol 2015;77:425-435.

22. Wade BJ. Spatial analysis of global prevalence of multiple sclerosis suggests need for an updated prevalence scale. Mult Scler Int 2014;2014: 124578.

23. Traboulsee A, Bass AD, Boster A, Berkovich R, Comi G, Fernández Ó, et al. Additional courses of alemtuzumab improved clinical and MRI outcomes in pooled CARE-MS I and II patients with disease activity after two courses: analysis of patients who received $\geq 3$ courses. Mult Scler 2018;24:517-518. 